\title{
CONCENTRATIONS DES PORTEURS ET CHAMP ÉLECTRIQUE LE LONG D'UN ÉCHANTILLON. ISOLANT OU SEMICONDUCTEUR. DÉVELOPPEMENTS DES SOLUTIONS STATIONNAIRES JUSQU'AU SECOND ORDRE. CAS DES FAIBLES POTENTIELS
}

\author{
R. MEAUDRE \\ Université de Lyon I, Laboratoire de Physique Electronique \\ 43, Bd du 11-Novembre 1918, 69-Villeurbanne
}

(Reçu le 13 avril 1972)

\begin{abstract}
Résumé. - Quand une différence de potentiel continue $V$ est appliquée à un isolant ou un semiconducteur muni d'électrodes bloquantes, des zones de charge d'espace s'établissent près de ces électrodes. La solution du problème, limitée au premier ordre en $V$ est connue ; dans certains cas, il peut être utile d'avoir cette solution développée jusqu'au second ordre, en particulier lors de la recherche de solutions dépendant du temps. C'est pourquoi dans cet article, les expressions développées jusqu'au second ordre en $V$, des densités de porteurs et du champ électrique en tout point de l'échantillon sont calculées.

Abstract. - When a dc potential difference $V$ is applied to an insulator or a semiconductor with two blocking electrodes, space charge takes place near the electrodes. The solution of this problem limited to the first order in $V$ is known, in many cases it may be useful to have this solution developed up to the second order, specially when we search time dependent solutions. Therefore in this paper the expressions of carrier densities and electric field in all the points of the sample, developed up to the second order in $V$, are calculated.
\end{abstract}

Introduction. - Dans un article récent [1], nous avons montré que, lors de la recherche de solutions dépendant du temps dans un isolant ou semiconducteur, des solutions du type $n(x, t)=c_{0}+n_{0}(x) n(t)$, $p(x, t)=c_{0}+p_{0}(x) p(t), E(x, t)=E_{0}(x) E(t)(n(x, t)$ $p(x, t)$ concentrations des porteurs, $E$ champ électrique, $x$ abscisse, $t$ temps) conduisent à la relation suivante :

$[n(t) p(\infty)-p(t) n(\infty)]\left[n_{0} \frac{\mathrm{d} p_{0}}{\mathrm{~d} x}-p_{0} \frac{\mathrm{d} n_{0}}{\mathrm{~d} x}\right]=0$

$n(\infty), p(\infty)$ expressions de $n(t)$ et $p(t)$ quand $t \rightarrow \infty$. La relation $n_{0} \frac{\mathrm{d} p_{0}}{\mathrm{~d} x}-p_{0} \frac{\mathrm{d} n_{0}}{\mathrm{~d} x}=0$ est satisfaite par les solutions stationnaires du premier ordre [2], il est important de savoir si elle l'est également par les solutions du second ordre, dans le cas contraire, l'éq. (1) impose l'autre relation

$$
n(t) p(\infty)-p(t) n(\infty)=0 .
$$

Dans cet article, nous nous proposons de calculer les expressions des densités de porteurs et du champ électrique développées jusqu'au second ordre.

Solutions stationnaires du second ordre. - 1 . SysTÈME D'ÉQUATIONS. - Le champ électrique et les concentrations de porteurs peuvent être obtenus en résolvant le système suivant :

$$
\begin{aligned}
P_{0}^{1 / 2}\left(\frac{\mu e}{2 \varepsilon D}\right)^{1 / 2} L & =F(\varphi, k)\left(\frac{L}{2}\right) \\
k^{\prime} \operatorname{tg} \varphi\left(\frac{L}{2}\right) & =\operatorname{sh} V^{*}
\end{aligned}
$$

$$
\begin{aligned}
(N- & \left.\frac{k_{2}}{k_{1}} P_{0}^{2}-P_{0}\right) L=P_{0}^{1 / 2}\left(\frac{8 \varepsilon D}{\mu e}\right)^{1 / 2} \times \\
& \times\left[-\delta(\varphi, k)+\operatorname{tg} \varphi\left(I-k^{2} \sin ^{2} \varphi\right)\right]_{\varphi(L / 2)} .
\end{aligned}
$$

Les hypothèses et notations sont celles utilisées dans les articles [2] et [3].

$P_{0}=n(0)=p(0)$ densité de porteurs au centre de l'échantillon.

$N$ densité de centres neutres, $L$ longueur de l'échantillon, $V^{*}=e V / 4 k T$.

$$
F(\varphi, k)=\int_{0}^{\varphi}\left(I-k^{2} \sin ^{2} t\right)^{-1 / 2} \mathrm{~d} t
$$

intégrale elliptique de première espèce.

$$
\mathcal{E}(\varphi, k)=\int_{0}^{\varphi}\left(I-k^{2} \sin ^{2} t\right)^{1 / 2} \mathrm{~d} t
$$

intégrale-elliptique de deuxième espèce. 
$E(x)=\left(E_{0} / \cos \varphi\right), E_{0}$ champ électrique au centre de l'échantillon, $k^{2}+k^{\prime 2}=I$.

La condition des faibles potentiels impose

$$
k^{\prime}(V) \rightarrow 0
$$

$\mathcal{E}(\varphi, k)$ et $F(\varphi, k)$ peuvent alors s'écrire :

$$
\begin{aligned}
& \mathcal{E}(\varphi, k)=\sin \varphi+ \frac{k^{\prime 2}}{4}\left[\log \operatorname{tg}\left(\frac{\varphi}{2}+\frac{\pi}{4}\right)-\sin \varphi\right] \\
& F(\varphi, k)=\log \operatorname{tg}\left(\frac{\varphi}{2}+\frac{\pi}{4}\right)-\frac{k^{\prime 2}}{4} \times \\
& \times\left[\frac{\sin \varphi}{\cos ^{2} \varphi}-\log \operatorname{tg}\left(\frac{\varphi}{2}+\frac{\pi}{4}\right)\right] .
\end{aligned}
$$

Les solutions du premier ordre sont obtenues avec l'approximation $p(0) \sim c_{0}, c_{0}$ étant la densité initiale de porteurs au centre; cherchons une expression de $p(0)$ de la forme

$$
p(0)=c_{0}[1+\alpha(V)] .
$$

Les relations (4), (5), (6) et la condition de neutralité initiale en tout point de l'échantillon permettent d'écrire :

$\alpha=$

$$
\frac{-\frac{I}{M} k^{\prime 2}\left[\frac{\sin \varphi}{\cos ^{2} \varphi}-\log \operatorname{tg}\left(\frac{\varphi}{2}+\frac{\pi}{4}\right)\right]_{\varphi(L / 2)}}{I+\frac{2 k_{2}}{k_{1}} c_{0}+\frac{k^{\prime 2}}{2 M}\left[\frac{\sin \varphi}{\cos ^{2} \varphi}-\log \operatorname{tg}\left(\frac{\varphi}{2}+\frac{\pi}{4}\right)\right]_{\varphi(L / 2)}}
$$

$$
M=\frac{L}{2 L_{D}}, \quad L_{D} \text { longueur de Debye }
$$

Nous devons alors trouver les développements de $\sin \varphi, \cos \varphi, \operatorname{tg} \varphi$ et $k^{\prime}$ en fonction de $V$.

2. DÉVeloppements DE $\sin \varphi, \cos \varphi, \operatorname{tg} \varphi, k^{\prime}$. Nous avons

$$
u=F(\varphi, k)=\frac{x}{L_{D}}\left(\frac{p(0)}{c_{0}}\right)^{1 / 2} .
$$

L'éq. (6) donne :

$$
\log \operatorname{tg}\left(\frac{\varphi}{2}+\frac{\pi}{4}\right)=u\left(I-\frac{k^{\prime 2}}{4}\right)+\frac{k^{\prime 2}}{4} \frac{\sin \varphi}{\cos ^{2} \varphi}
$$

c'est-à-dire :

$$
\begin{aligned}
& \cos \varphi=\left\{\operatorname{ch}\left[u\left(I-\frac{k^{\prime 2}}{4}\right)+\frac{k^{\prime 2}}{4} \frac{\sin \varphi}{\cos ^{2} \varphi}\right]\right\}^{-1} \\
& \sin \varphi=\operatorname{th}\left[u\left(I-\frac{k^{\prime 2}}{4}\right)+\frac{k^{\prime 2}}{4} \frac{\sin \varphi}{\cos ^{2} \varphi}\right] .
\end{aligned}
$$

Les développements peuvent alors s'écrire :

$\cos \varphi=(\operatorname{ch} u)^{-1}\left[I-\frac{k^{\prime 2}}{4}\left(\frac{\sin \varphi}{\cos ^{2} \varphi}\right.\right.$ th $u-u$ th $\left.\left.u\right)\right]$

$\sin \varphi=\operatorname{th} u\left[I-\frac{k^{\prime 2}}{4}(\operatorname{th} u-\operatorname{coth} u) \times\right.$

$$
\left.\times\left(\frac{\sin \varphi}{\cos ^{2} \varphi}-u\right)\right]
$$

$\operatorname{tg} \varphi=\operatorname{sh} u\left[I-\frac{k^{\prime 2}}{4} \operatorname{coth} u\left(u-\frac{\sin \varphi}{\cos ^{2} \varphi}\right)\right]$.

Le remplacement de $(\cos \varphi)^{-1} \operatorname{par}\left(I+\operatorname{tg}^{2} \varphi\right)^{1 / 2}$ dans l'éq. (10) permet d'obtenir une équation du quatrième degré en $\operatorname{tg} \varphi$, donc en principe $\operatorname{tg} \varphi$. Le processus étant compliqué, nous prendrons en première approximation, l'expression de $\cos \varphi$ correspondant à $k^{\prime}=0$ c'est-à-dire $\cos \varphi=(\operatorname{ch} u)^{-1}$. Nous avons alors :

$$
\operatorname{tg} \varphi=\operatorname{sh} u\left[I-\frac{k^{\prime 2}}{4}\left(u \operatorname{coth} u-\operatorname{ch}^{2} u\right)\right] .
$$

Les éq. (3) et (10) permettent de calculer $k^{\prime}$ :

$$
k^{\prime}=\left.\left(V^{*}+\frac{V^{* 3}}{3 !}\right)(\operatorname{sh} u)^{-1}\right|_{u(L / 2)}
$$

3. CAlCul DE $\alpha$.

$$
u\left(\frac{L}{2}\right)=\frac{L}{2 L_{D}}\left(\frac{p(0)}{c_{0}}\right)^{1 / 2} \simeq M\left(I+\frac{\alpha}{2}\right) .
$$

Lorsque $M \alpha / 2 \ll I$ nous avons

$$
\begin{aligned}
\operatorname{ch} u\left(\frac{L}{2}\right) & =\operatorname{ch} M\left(I+\frac{M \alpha}{2} \operatorname{th} M\right) \\
\operatorname{sh} u\left(\frac{L}{2}\right) & =\operatorname{sh} M\left(I+\frac{M \alpha}{2} \operatorname{coth} M\right) \\
\operatorname{coth} u\left(\frac{L}{2}\right) & =\operatorname{coth} M\left[I+\frac{M \alpha}{2}(\operatorname{th} M-\operatorname{coth} M)\right] .
\end{aligned}
$$

De l'expression (8) nous tirons :

$$
\begin{aligned}
\alpha\left\{I+\frac{2 k_{2}}{k_{1}} c_{0}+\frac{V^{* 2}}{2 M}\left[\operatorname{coth} M-\frac{M}{\operatorname{sh}^{2} M}+\right.\right. \\
+M \operatorname{coth} M\left(\operatorname{th} M-\operatorname{coth} M-\frac{I}{\operatorname{ch} M \operatorname{sh} M}\right. \\
\left.\left.\left.+\frac{2 M}{\operatorname{sh}^{2} M}\right)\right]\right\}=-\frac{V^{* 2}}{M}\left(\operatorname{coth} M-\frac{M}{\operatorname{sh}^{2} M}\right) .
\end{aligned}
$$

Les cas expérimentaux correspondent à $M \gtrsim 1$ donc :

$$
\alpha=\frac{-\frac{V^{* 2}}{M}\left(\operatorname{coth} M-\frac{M}{\operatorname{sh}^{2} M}\right)}{I+\frac{2 k_{2}}{k_{1}} c_{0}} .
$$


4. Calculs DE $n(x), p(x)$ ET $E(x)$. - Les relations $E(x) \sim E_{0} \operatorname{ch} u, \log \frac{p(x)}{p(0)}=\log \frac{n(0)}{n(x)}=$

$$
=\frac{e}{k T} \int_{0}^{x} E \mathrm{~d} x, V=\int_{-L / 2}^{L / 2} E \mathrm{~d} x
$$

et l'éq. (7) permettent finalement d'obtenir :

$n(x)=c_{0}\left\{I+\alpha-2 V^{*} \frac{\operatorname{sh} \frac{x}{L_{D}}}{\operatorname{sh} M}+2 V^{* 2}\left(\frac{\operatorname{sh} \frac{x}{L_{D}}}{\operatorname{sh} M}\right)^{2}\right\}$
$p(x)=c_{0}\left\{I+\alpha+2 V^{*} \frac{\operatorname{sh} \frac{x}{L_{D}}}{\operatorname{sh} M}+2 V^{* 2}\left(\frac{\operatorname{sh} \frac{x}{L_{D}}}{\operatorname{sh} M}\right)^{2}\right\}$

$E(x)=\frac{V}{L} \frac{M}{\operatorname{sh} M} \operatorname{ch} \frac{x}{L_{D}}$.
Ces expressions sont valables lorsque $V^{*} \lesssim I$; elles ne permettent pas de remplir la condition

$$
n_{0} \frac{\mathrm{d} p_{0}}{\mathrm{~d} x}-p_{0} \frac{\mathrm{d} n_{0}}{\mathrm{~d} x}=0
$$

Conclusion. - En utilisant les développements des intégrales elliptiques de première et deuxième espèces, nous avons calculé les expressions de $n(x), p(x)$, et $E(x)$ développées jusqu'au second ordre en $V$. Ce résultat est important car, lors de la recherche de solutions dépendant du temps, il impose la solution $n(t) p(\infty)=p(t) n(\infty)$. Nous avons discuté les conséquences de ceci dans un article récent [1], le fait essentiel étant que les solutions du type

$$
\begin{aligned}
& n(x, t)=c_{0}+n_{0}(x) n(t), \\
& p(x, t)=c_{0}+p_{0}(x) p(t)
\end{aligned}
$$

sont incompatibles avec l'hypothèse du blocage total des porteurs aux électrodes.

\section{Bibliographie}

[1] Meaudre (R.) et Mesinard (G.), proposé à la Revue de Physique Appliquée.

[2] Huck (J.) et Maitrot (M.), J. Physique, 1965, 26, 229.

[3] Huck (J.) et Maitrot (M.), J. Physique, 1966, 27, 422. 